\title{
Three-dimensional adaptive soft phononic crystals
}

\section{Citation}

Babaee, Sahab, Pai Wang, and Katia Bertoldi. 2015. "Three-Dimensional Adaptive Soft Phononic Crystals." Journal of Applied Physics 117 (24): 244903. https://doi.org/10.1063/1.4923032.

\section{Permanent link}

http://nrs.harvard.edu/urn-3:HUL.InstRepos:41461228

\section{Terms of Use}

This article was downloaded from Harvard University's DASH repository, and is made available under the terms and conditions applicable to Other Posted Material, as set forth at http:// nrs.harvard.edu/urn-3:HUL.InstRepos:dash.current.terms-of-use\#LAA

\section{Share Your Story}

The Harvard community has made this article openly available.

Please share how this access benefits you. Submit a story.

Accessibility 


\title{
Three-dimensional adaptive soft phononic crystals
}

\author{
Sahab Babaee, ${ }^{1}$ Pai Wang, ${ }^{1}$ and Katia Bertoldi ${ }^{1,2}$ \\ ${ }^{1}$ School of Engineering and Applied Sciences, Harvard University, Cambridge, Massachusetts 02138, USA \\ ${ }^{2}$ Kavli Institute, Harvard University, Cambridge, Massachusetts 02138, USA
}

(Received 29 April 2015; accepted 12 June 2015; published online 25 June 2015)

\begin{abstract}
We report a new class of three-dimensional (3D) adaptive phononic crystals whose dynamic response is controlled by mechanical deformation. Using finite element analysis, we demonstrate that the bandgaps of the proposed 3D structure can be fully tuned by the externally applied deformation. In fact, our numerical results indicate that the system acts as a reversible phononic switch: a moderate level of applied strain (i.e., -0.16) is sufficient to completely suppress the bandgap, and upon the release of applied strain, the deformed structure recovers its original shape, which can operate with a sizable bandgap under dynamic loading. In addition, we investigate how material damping significantly affects the propagation of elastic waves in the proposed 3D soft phononic crystal. We believe that our results pave the way for the design of a new class of soft, adaptive, and re-configurable $3 \mathrm{D}$ phononic crystals, whose bandgaps can be easily tuned and switched on/off by controlling the applied deformation. (C) 2015 AIP Publishing LLC.
\end{abstract}

[http://dx.doi.org/10.1063/1.4923032]

\section{INTRODUCTION}

Three-dimensional (3D) periodic structures are attracting significant interest not only for their remarkable static properties $^{1-4}$ but also for their ability to control the propagation of elastic waves through bandgaps, i.e., frequency ranges of strong wave attenuation. ${ }^{5,6}$ Such bandgaps can be formed either by Bragg scattering ${ }^{7-9}$ or by exploiting local resonance within the medium. ${ }^{10-13}$ Bragg-type bandgaps are intrinsic to order and typically occur at wavelengths of the order of the unit cell size, whereas locally resonant bandgaps arise in the vicinity of the natural frequency of the resonating units and are less affected by the spatial periodicity of the system.

While most of the proposed 3D phononic crystals operate in fixed ranges of frequencies that are impractical to tune and control after the assembly; ${ }^{14-19}$ it has been shown that the dynamic responses of structures could be altered by mechanically deforming them. ${ }^{20-22}$ However, a large amount of loading is typically required to significantly affect the position and width of the bandgaps.

It has been recently demonstrated that the tunability of 2D phononic crystals can be significantly enhanced by triggering mechanical instabilities along the loading path. ${ }^{23-26}$ In fact, the instabilities may induce dramatic homogeneous and reversible changes of the geometry that can be exploited not only to alter but also to turn on and off the bandgaps even under moderate levels of applied deformation. However, although recent studies have shown that buckling can be an effective way to achieve better tunability of $2 \mathrm{D}$ phononic devices, ${ }^{26-28}$ no similar studies have been reported for $3 \mathrm{D}$ systems.

In this study, we focus on a 3D soft crystal consisting an array of elastomeric patterned spherical shells (see Fig. 1). This structure has been recently shown to undergo an instability under uniaxial compression which results in folding of all the building blocks and macroscopic auxetic behavior. ${ }^{29}$
Here, we investigate numerically the effect of the applied compression on the propagation of small-amplitude elastic waves, aiming to the design of a $3 \mathrm{D}$ reconfigurable phononic crystal with enhanced tunability. To this end, first we identify the geometric parameters leading to the widest bandgap in the undeformed configuration and then investigate the effect of the applied compression on the propagation of elastic waves. Finally, since elastomeric materials are dissipative, we study how material damping affects the dynamic response of the system.

\section{3D PHONONIC CRYSTAL}

The 3D structure investigated in this study is composed of a periodic array of structured shells arranged to form a body centered cubic $(b c c)$ crystal. Each spherical shell is patterned with a regular array of six circular voids and is fully characterized by two design parameters: ${ }^{29,30}$ the angle $\alpha$ that defines the narrowest width of the ligament, and the ratio $\tau=w_{i} / t$ between the inner narrowest width of the ligaments and the radial shell thickness (see Fig. 1).

The response of our 3D structure is conveniently captured using the unit cell shown in Fig. 1. Such unit cell is a cube with edges $a=4 r_{o} / \sqrt{3}$ ( $r_{o}$ is the outer radius of the spherical shell) and comprises two structured shells-one full shell at the center with eight one-eighths attached to it.

Finally, the porosity of the 3D structure can be calculated as

$$
\psi=1-\frac{2 V_{\text {shell }}}{V_{\text {unit }}}
$$

where $V_{\text {unit }}=a^{3}$ is the volume of the cubic unit cell, $V_{\text {shell }}$ is the volume fraction of the structured shell, and the pre-factor 2 is the number of structured shells in each unit cell. Note that $V_{\text {shell }}$ is simply obtained by subtracting the volume of the six voids from the volume of the intact shell 

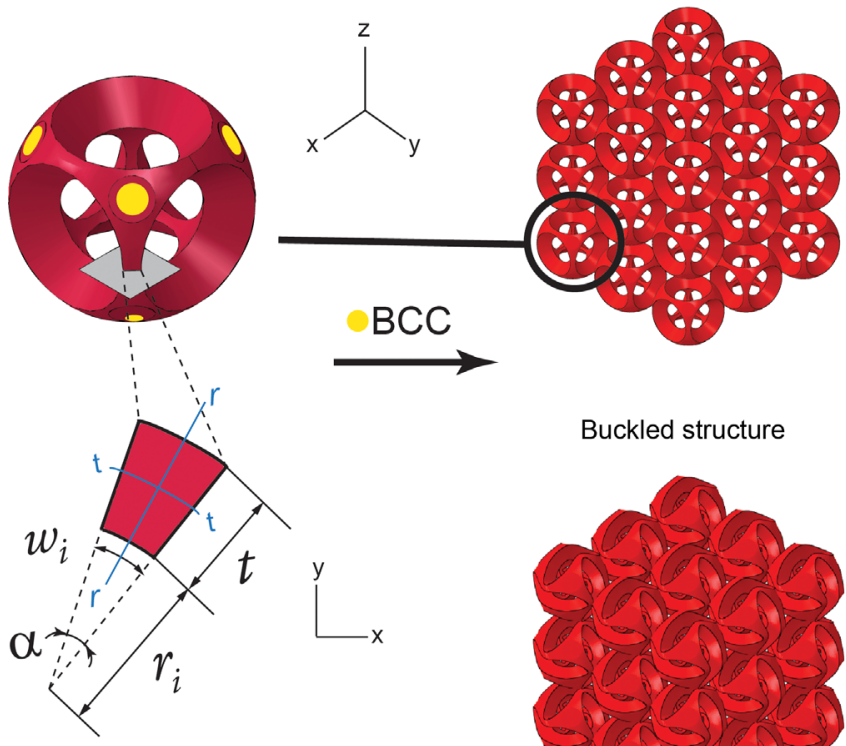

Cross-sectional view

$$
V_{\text {shell }}=V_{\text {intact shell }}-6 V_{\text {void }},
$$

where

$$
V_{\text {intact shell }}=\frac{4}{3} \pi\left(r_{o}^{3}-r_{i}^{3}\right)
$$

and

$$
V_{\text {void }}=\frac{2}{3} \pi\left(r_{o}^{3}-r_{i}^{3}\right)[1-\cos (\pi / 4-\alpha / 2)],
$$

$r_{i}$ denoting the inner radius of the spherical shell. ${ }^{30}$ Combining Eqs. (1)-(4), the porosity of the 3D crystal can be obtained as

$$
\psi=1-\frac{8 \pi\left(r_{o}^{3}-r_{i}^{3}\right)[-2+3 \cos (\pi / 4-\alpha / 2)]}{3\left(4 r_{0} / \sqrt{3}\right)^{3}}
$$

\section{GENERAL FORMULATION}

Here, we present the governing equations and material model, which are employed in the numerical calculations.

\section{A. Governing equations}

The deformation of the 3D phononic crystal is described by the deformation gradient

$$
\mathbf{F}=\frac{\partial \mathbf{x}}{\partial \mathbf{x}_{0}}
$$

mapping a point in the material from the reference position $\mathbf{x}_{0}$ to its current location $\mathbf{x}$. The material is assumed to be hyperelastic, characterized by a strain energy function $W=W(\mathbf{F})$, which is defined in the reference configuration. The first Piola-Kirchhoff stress $\mathbf{S}$ is thus related to the deformation gradient $\mathbf{F}$ by
Undeformed unit cell

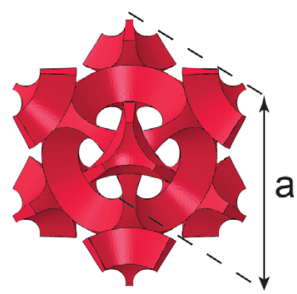

Buckled unit cell

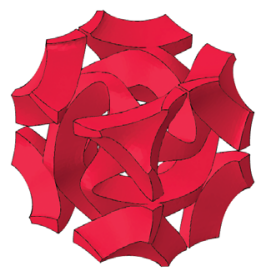

$$
\mathbf{S}=\frac{\partial W}{\partial \mathbf{F}}
$$

In the absence of body forces, the equation of motions in the reference configuration can be written as

$$
\operatorname{Div} \mathbf{S}=\rho_{0} \frac{D^{2} \mathbf{x}}{D t^{2}}
$$

where Div represents the divergence operator in the undeformed/reference configuration, $D / D t$ is the material time derivative, and $\rho_{0}$ denotes the reference mass density.

To investigate the effect of the applied deformation on the propagation of small-amplitude elastic waves, incremental deformations superimposed upon a given state of finite deformation are considered. Denoting with $\dot{\mathbf{S}}$ the increment of the first Piola-Kirchhoff stress, the incremental forms of the governing equations are given by

$$
\operatorname{Div} \dot{\mathbf{S}}=\rho_{0} \frac{D^{2} \dot{\mathbf{x}}}{D t^{2}}
$$

where $\dot{\mathbf{x}}$ denotes the incremental displacements. Furthermore, linearization of the constitutive equation (7) yields

$$
\dot{\mathbf{S}}=\mathbb{L}: \dot{\mathbf{F}}, \text { with } \mathbb{L}_{i j k l}=\frac{\partial^{2} W}{\partial F_{i j} \partial F_{k l}},
$$

where $\dot{\mathbf{F}}$ denotes the incremental deformation gradient.

The incremental boundary value problem is often formulated in an updated Lagrangian formulation, where the deformed state is used as the reference configuration for the calculation of the incremental quantities. ${ }^{31}$ Push-forward transformations allow the introduction of the incremental updated stress quantity $\boldsymbol{\Sigma}$ given by

$$
\Sigma=\frac{1}{J} \dot{\mathbf{S}} \mathbf{F}^{T}
$$


For a non-linear elastic material, the incremental constitutive equation takes the linear form

$$
\Sigma=\mathbb{C}: \operatorname{grad} \mathbf{u}
$$

to the first order, where grad denotes the gradient operator in the deformed/current configuration, $\mathbf{u} \doteq \dot{\mathbf{x}}$ and

$$
\mathbb{C}_{i j k l}=J^{-1} F_{j n} F_{l q} \frac{\partial^{2} W}{\partial F_{i n} \partial F_{k q}} .
$$

Finally, the incremental equations of motions take the form

$$
\operatorname{div} \boldsymbol{\Sigma}=\rho \frac{d^{2} \mathbf{u}}{d t^{2}}
$$

where div represents the divergence operator in the deformed/current configuration, $\rho$ denotes the current material density, and $d / d t$ is the spatial time derivative.

Here, we focus on the propagation of small-amplitude elastic waves defined by

$$
\mathbf{u}(\mathbf{x}, t)=\tilde{\mathbf{u}}(\mathbf{x}) \exp (-i \omega t),
$$

where $\omega$ is the angular frequency of the propagating wave and $\tilde{\mathbf{u}}$ denotes the magnitude of the incremental displacement. It follows from (12) that

$$
\boldsymbol{\Sigma}(\mathbf{x}, t)=\tilde{\Sigma}(\mathbf{x}) \exp (-i \omega t),
$$

so that equations (14) become

$$
\operatorname{div} \tilde{\Sigma}=\rho \omega^{2} \tilde{\mathbf{u}},
$$

which represent the frequency-domain wave equations.

\section{B. Material model}

The 3D phononic crystal is assumed to be made of silicon rubber. The response of such material is captured using a nearly incompressible Neo-Hookean model, ${ }^{32}$ whose strain energy density is given by

$$
W\left(I_{1}, J\right)=\frac{G}{2}\left(I_{1}-3\right)-G \log (J)+\frac{K}{2}(J-1)^{2},
$$

where $I_{1}=\operatorname{trace}\left(\mathbf{F}^{T} \mathbf{F}\right), J=\operatorname{det}(\mathbf{F})$ and $\mathbf{F}$ is the deformation gradient. Moreover, $G$ and $K$ are the initial shear and bulk moduli of the material, respectively.

Substituting (18) into (7) and (10), the nominal stress $\mathbf{S}$ and the incremental modulus $\mathbb{L}$ are obtained as

$$
\mathbf{S}=G \mathbf{F}+[K J(J-1)-G] \mathbf{F}^{-T},
$$

and

$$
\begin{aligned}
\mathbb{L}= & G \mathbb{I}+K J(2 J-1) \mathbf{F}^{-T} \otimes \mathbf{F}^{-T} \\
& +[K J(J-1)-G] \mathbb{I}^{T},
\end{aligned}
$$

where

$$
\mathbb{I}_{i j k l}=\frac{\partial F_{k l}}{\partial F_{i j}}=\delta_{i k} \delta_{j l} .
$$

In this study, we assume that the $3 \mathrm{D}$ phononic crystal is made of silicon-based rubber (Elite Double 32:Zhermack), which is characterized by $G=0.27 \mathrm{MPa}, K=13.41 \mathrm{MPa}$, and density $\rho=965 \mathrm{Kg} \mathrm{m}^{-3}$. $^{29}$

\section{NUMERICAL PROCEDURE AND IMPLEMENTATION}

In this section, we first present the analysis performed to study the dynamic response of 3D phononic crystals of infinite size, accounting for the effect of the applied predeformation. Then, we describe how we study the effect of material damping on the propagation of elastic waves.

All numerical simulations are performed using the commercial finite element package Abaqus/Standard (SIMULIA, Providence, RI). The 3D models are constructed using quadratic brick elements (element type C3D10) and the accuracy of the mesh is insured by a mesh refinement study.

\section{A. Wave propagation analysis in 3D adaptive phononic crystals}

The analysis conducted to investigate the propagation of elastic waves in 3D deformable phononic crystals of infinite size consists of three steps: (i) first, the stability of the structure is investigated through a linear perturbation analysis; ${ }^{33,34}$ (ii) then, the non-linear post-buckling response of the system is simulated by using periodic boundary conditions and introducing a small imperfection on the initial geometry in the form of the critical mode; ${ }^{34}$ (iii) finally, the propagation of small-amplitude elastic waves at a given level of deformation is investigated by solving the frequencydomain wave equation.

Focusing on step (iii), we discretize the frequencydomain wave equations (17) using the Galerkin finite element method and obtain an algebraic eigenvalue problem in matrix form

$$
\left[\mathbf{K}_{(n \times n)}-\omega^{2} \mathbf{M}_{(n \times n)}\right] \mathbf{U}_{(n \times 1)}=0
$$

where $\mathbf{K}$ and $\mathbf{M}$ are the global mass and stiffness matrices, $\mathbf{U}$ is the nodal displacement vector, and $n$ is the total number of degrees of freedom (i.e., number of nodes multiply by degrees of freedom per node).

By virtue of Bloch's theorem, ${ }^{35}$ the following relationship between the displacements of two nodes, $A$ and $B$, periodically located on the outer faces of the unit cell is obtained:

$$
\mathbf{u}_{A}=\mathbf{u}_{B} \exp \left(i \mathbf{k} \cdot \mathbf{r}_{A B}\right)
$$

where $\mathbf{u}_{A}$ and $\mathbf{u}_{B}$ denote the displacement of nodes $A$ and $B$, respectively, and $\mathbf{r}_{A B}$ is the distance between the nodes $A$ and $B$ in the deformed configuration. Moreover, $\mathbf{k}$ is the Blochwave vector that can be expressed in terms of the reciprocal lattice vectors $\mathbf{b}_{i}$

$$
\begin{aligned}
& \mathbf{b}_{1}=2 \pi \frac{\mathbf{a}_{2} \times \mathbf{a}_{3}}{\|\mathbf{z}\|^{2}}, \\
& \mathbf{b}_{2}=2 \pi \frac{\mathbf{a}_{3} \times \mathbf{a}_{1}}{\|\mathbf{z}\|^{2}}, \\
& \mathbf{b}_{3}=2 \pi \frac{\mathbf{a}_{1} \times \mathbf{a}_{2}}{\|\mathbf{z}\|^{2}},
\end{aligned}
$$


where $\mathbf{a}_{1}, \mathbf{a}_{2}$, and $\mathbf{a}_{3}$ are the lattice vectors spanning the unit cell (see Fig. 2) and $\mathbf{z}=\mathbf{a}_{1} \cdot\left(\mathbf{a}_{2} \times \mathbf{a}_{3}\right)$. Note that when the Bloch-type boundary conditions (23) are incorporated into Eq. (22), the stiffness matrix becomes a function of $\mathbf{k}$ (i.e., $\mathbf{K}=\mathbf{K}(\mathbf{k})$ ). Therefore, to obtain the dispersion relations $\omega=\omega(\mathbf{k})$, the eigenvalue problem specified by Eq. (22) needs to be solved for a number of wave vectors $\mathbf{k}$.

Since the reciprocal lattice is also periodic, we can restrict the wave vectors $\mathbf{k}$ to a certain region of the reciprocal space called the first Brillouin zone (dark grey cube in Fig. 2(b)). ${ }^{36}$ In addition, we may further reduce the domain taking advantage of its reflectional and rotational symmetries. The reduced domain is referred to the irreducible Brillouin zone (IBZ) (red pyramid GXMR in Fig. 2(b)). ${ }^{37}$

Next, we show that Eq. (22) can be solved using either the full direct method or the Reduced Bloch Mode Expansion (RBME) method ${ }^{38}$ to significantly reduce the computational cost.

\section{Full (direct) method}

Here, we solve Eq. (22) for a large number of $\mathbf{k}$ vectors on the perimeter of IBZ (i.e., G-X-M-G-R-M-X-R path in Fig. 3) to obtain the dispersion relations $\omega=\omega(\mathbf{k})$. In particular, we use ten uniformly spaced k-points on each edge of the IBZ shown in Fig. 3. Given the large sizes of the mass and stiffness matrices involved in the calculations (i.e., $n \simeq$ 200000 for our unit cells), this method is computationally very expensive.

\section{RBME method}

To reduce the size of the problem and consequently the computational cost, we employ a reduced basis composed of a selected set of Bloch eigenvectors. ${ }^{38}$ To this end, we use a linear transformation given by (a)
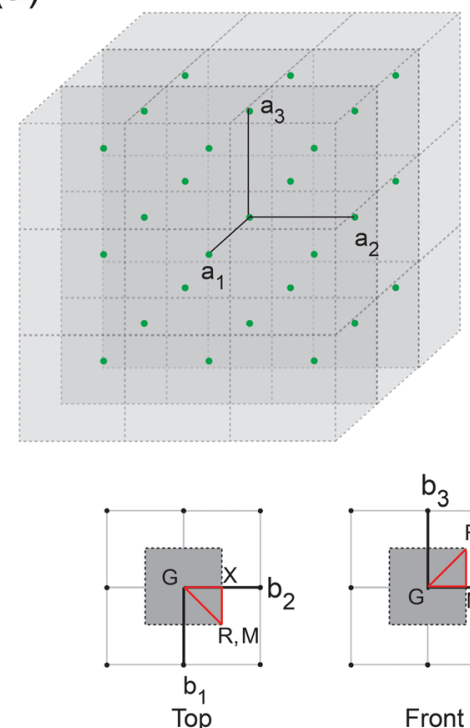
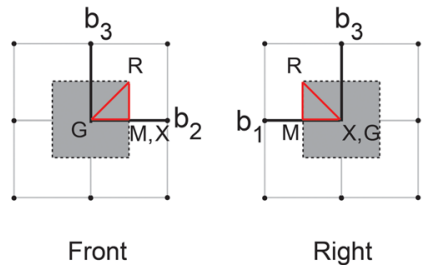

FIG. 2. Schematic of point lattice (green dots in (a)), reciprocal lattice (black dots in (b)), first Brillouin zone (grey cube in (b)), and irreducible Brillouin zone (red pyramid GXMR in (b)). Three different views of the reciprocal lattice are shown at the bottom.

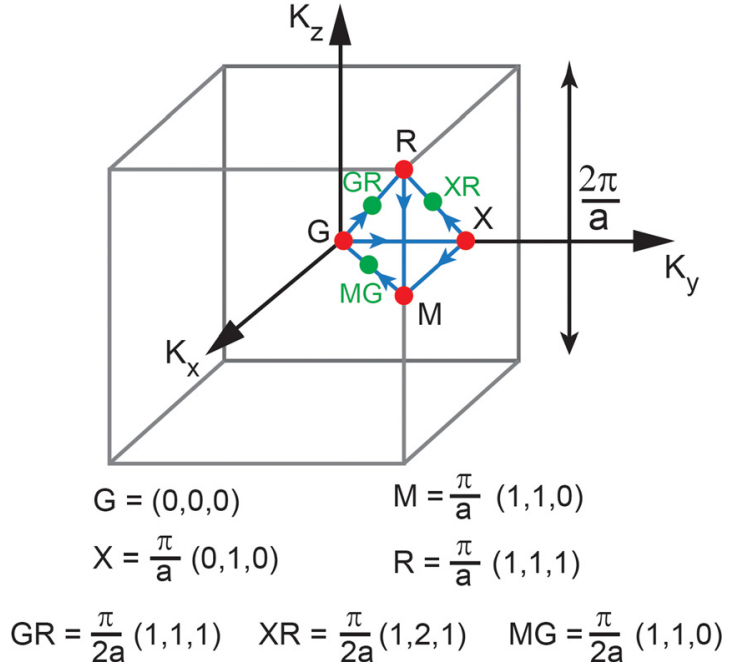

FIG. 3. First Brillouin zone (cube of side $2 \pi / a$ ) and IBZ with the seven high-symmetry points used in this study: corner points $(\mathrm{G}, \mathrm{X}, \mathrm{M}, \mathrm{R})$ and points on mid-diagonal lines (GR, XR, MG).

$$
\mathbf{U}_{(n \times 1)}=\phi_{(n \times m)} \mathbf{V}_{(m \times 1)},
$$

where $\mathbf{U}_{(n \times 1)}$ is the nodal Bloch displacement vector, $\mathbf{V}_{(m \times 1)}$ is the reduced Bloch displacement vector, $\phi_{(n \times m)}$ is the transformation matrix, $n$ is the total number of degrees of freedom, and $m(m \ll n)$ is the total number of Bloch modes in the reduced basis. Combining (25) and (22) and premultiplying by the complex conjugate transpose $\phi_{(m \times n)}^{\star}$ yields the eigenvalue problem in the reduced basis

$$
\begin{aligned}
& {\left[\phi_{(m \times n)}^{\star} \mathbf{K}(\mathbf{k})_{(n \times n)} \phi_{(n \times m)}\right.} \\
& \left.\quad-\omega^{2} \phi_{(m \times n)}^{\star} \mathbf{M}_{(n \times n)} \phi_{(n \times m)}\right] \mathbf{V}_{(m \times 1)}=0,
\end{aligned}
$$

which can be re-express as

$$
\left[\overline{\mathbf{K}}(\mathbf{k})_{(m \times m)}-\omega^{2} \overline{\mathbf{M}}_{(m \times m)}\right] \mathbf{V}_{(m \times 1)}=0, \quad m \ll n,
$$

where $\overline{\mathbf{K}}$ and $\overline{\mathbf{M}}$ are the reduced stiffness and mass matrices

$$
\begin{aligned}
\overline{\mathbf{K}} & =\phi_{(m \times n)}^{\star} \mathbf{K}(\mathbf{k})_{(n \times n)} \phi_{(n \times m)}, \\
\overline{\mathbf{M}} & =\phi_{(m \times n)}^{\star} \mathbf{M}_{(n \times n)} \phi_{(n \times m)} .
\end{aligned}
$$

The reduced basis used in this study is composed of Bloch eigenvectors evaluated at seven high-symmetry points: points $\mathrm{G}, \mathrm{X}, \mathrm{M}$, and $\mathrm{R}$ (corners of the IBZ) and GR, $\mathrm{XR}$, and MG shown in Fig. 3. The choice of these points is motivated by both the crystal structure and group theory. ${ }^{38}$ To construct the transformation matrix $\phi_{(n \times m)}$, we solve (22) only at the selected seven high-symmetry points and assemble the corresponding eigenvectors in column-wise form as

$$
\phi_{(n \times m)}=\left[\phi_{G} \phi_{X} \phi_{M} \phi_{R} \phi_{M G} \phi_{G R} \phi_{X R}\right] .
$$

Furthermore, since in this study we are interested in computing the first 160 dispersion branches, we find that 800 eigenmodes calculated at each of the seven high-symmetry points are sufficient for the band structure calculations to be accurate up to the range of interest. The accuracy of the band 
structure calculated by employing the RBME method with seven high-symmetry points is assured by reproducing the same band diagrams calculated via the full (direct) method, as shown in Fig. 4 for a 3D phononic crystal characterized by $(\alpha, \tau)=(0.03,0.2)$. Note that when using the RBME method, the sizes of the mass and stiffness matrices are reduced from $n \simeq 200000$ to $m=5600$, significantly reducing the computational cost.

\section{B. Wave propagation analysis in 3D damped phononic crystals}

To study the effect of material dissipation on the propagation of elastic waves, we employ Rayleigh (proportional) damping model ${ }^{39,40}$ and define a damping matrix $\mathbf{C}$ linearly proportional to the mass and stiffness matrices $\mathbf{M}$ and $\mathbf{K}(\mathbf{k})$

$$
\mathbf{C}(\mathbf{k})=p \mathbf{M}+q \mathbf{K}(\mathbf{k}),
$$

where $p$ and $q$ are the mass and stiffness proportional damping coefficients $(p, q \geq 0)$. The discretized finite element frequency-domain wave equations of the damped phononic crystal are then given by

$$
\left[\mathbf{K}(\mathbf{k})+i \omega \mathbf{C}(\mathbf{k})-\omega^{2} \mathbf{M}\right] \mathbf{U}=0,
$$

and the wave-vector-dependent damping ratio $\xi_{i}(\mathbf{k})$ for the $i$ th branch of the damped band structure is obtained as ${ }^{41}$

$$
\xi_{i}(\mathbf{k})=\frac{p+q \omega_{i}^{2}(\mathbf{k})}{2 \omega_{i}(\mathbf{k})},
$$

where $\omega_{i}(\mathbf{k})$ denotes the $i$-th frequency obtained in the absence of damping. Since for underdamped conditions (i.e., $\xi_{i}<1$ ), the effect of the damping parameter $p$ in Eq. (30) is negligible, ${ }^{42,43}$ we consider a stiffness-proportional Raleigh damping matrix (i.e., $p=0$ and $q \neq 0$ ) in all the calculations and report all results in terms of the dimensionless damping coefficient $\bar{q}=q c_{T} / a$. We also note that for elastomers $\xi_{i}=q \omega_{i} / 2=\pi \bar{q} \bar{f} \sim 0.05,{ }^{44-46}$ so that if we focus on the upper limit of the bandgap (i.e., $\bar{f}_{\text {upper }} \sim 0.75$ ), we obtain $\bar{q} \sim 0.02$ for elastomeric materials.

To investigate the propagation of elastic waves in a dissipative medium, we construct 3D models of finite size comprising different numbers of unit cells and calculate their steady-state dynamic linearized response to harmonic excitations for different levels of material damping. In this set of analyses, we account for energy dissipation by directly introducing Raleigh damping in the simulations (i.e., the *DAMPING keyword is used to specify Raleigh damping parameters as material properties in Abaqus/ Standard $\left.{ }^{47}\right)$. Moreover, we excite the structure by applying an harmonic displacement $\left(A_{\text {in }} \cos (\omega t)\right)$ to the central node of one of its faces at one end and calculate the displacement of the corresponding node on the opposite face at the other end $\left(A_{\text {out }} \cos (\omega t)\right)$ over the frequency ranges of interest. The transmission spectrum $(\mathrm{dB})$ is then computed as $10 \log \left(A_{\text {out }}(\omega) / A_{\text {in }}(\omega)\right)^{2}$.

\section{RESULTS}

\section{A. Elastic waves propagation in the undeformed phononic crystal}

We start by investigating the propagation of elastic waves in undeformed phononic crystals of infinite size. In Fig. 5, we report the band diagram for crystals characterized by $(\alpha, \tau)=(0.14,0.2),(0.14,1.0)$, and $(0.03,0.2)$. In all these diagrams, the non-dimensional frequency $\bar{f}=\omega a /\left(2 \pi c_{T}\right)$ is plotted versus a set of wave vectors $\mathbf{k}$ spanning the perimeter of the IBZ shown in Fig. 3. Here, $c_{T}=\sqrt{G / \rho}$ is the speed of transverse elastic waves propagating within the homogeneous bulk material and $a$ is the characteristic size of the unit cell in the undeformed configuration (see Fig. 1). As expected, we find that the microstructural design parameters $\alpha$ and $\tau$ strongly affect the existence and width of the bandgap. In fact, while the 3D phononic crystals with $(\alpha, \tau)$ $=(0.14,0.2)$ and $(0.14,1.0)$ have no frequency ranges of strong wave attenuation, the structure with $(\alpha, \tau)=(0.03,0.2)$ has a bandgap for $\bar{f}=0.52-0.72$. It is important to note that this is a Bragg-type bandgap, since it is very sensitive to the periodicity and geometry of the crystal. To demonstrate this, in Fig. 6 we report the band structure for a phononic crystal characterized exactly by the same design parameters (i.e., $(\alpha, \tau)=(0.03,0.2))$, but with the structured shells arranged differently and attached to each other through the thin
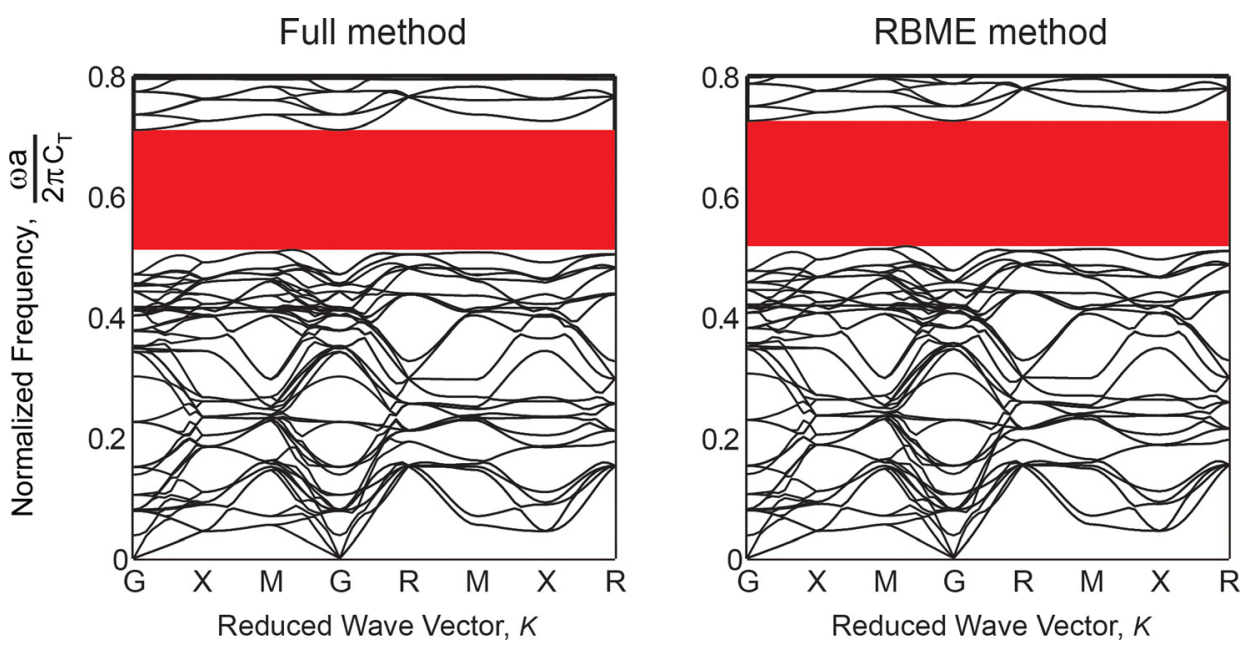

FIG. 4. Band diagrams for a 3D phononic crystal characterized by $(\alpha, \tau)$ $=(0.03,0.2)$ calculated using full (direct) method (left) and the RBME method (right). 


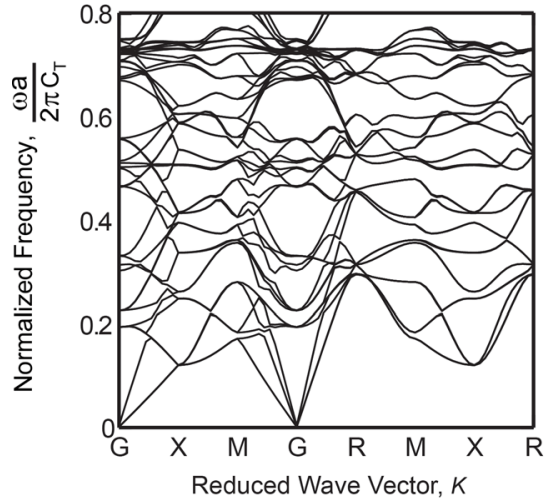

$\alpha=0.14 \mathrm{rads}$

$$
\tau=\frac{1}{1}=1
$$

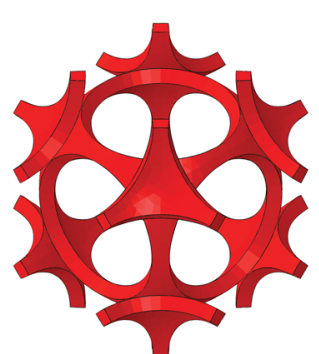

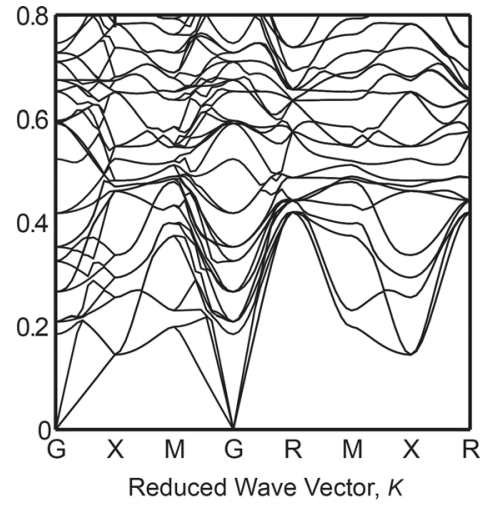

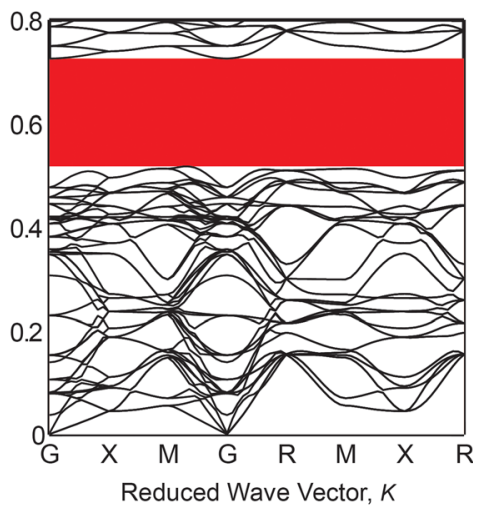

$\alpha=$ const.

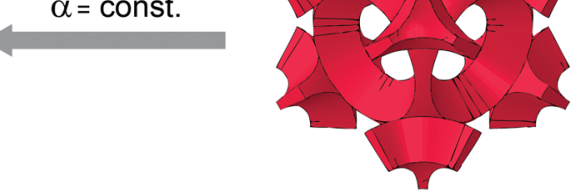

$\tau=$ const.

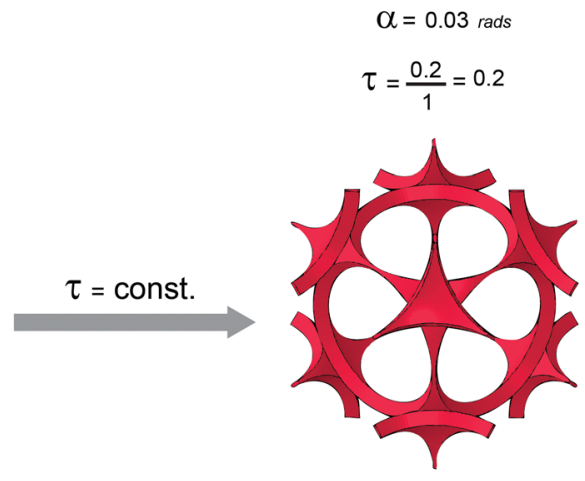

FIG. 5. Effect of design parameters $\alpha$ and $\tau$ on the band structures. In the dispersion relation plots, the non-dimensional frequency $\bar{f}=\omega a / 2 \pi c_{T}$ is plotted versus the wave vectors $\mathbf{k}$. Three different configurations are considered characterized by $(\alpha, \tau)=(0.14,0.2)$ (center), $(0.14,1.0)$ (left), and $(0.03,0.2)$ (right). The shaded red region in the right plot highlights the bandgap, i.e., ranges of frequencies for which the elastic waves are not allowed to propagate along any direction.
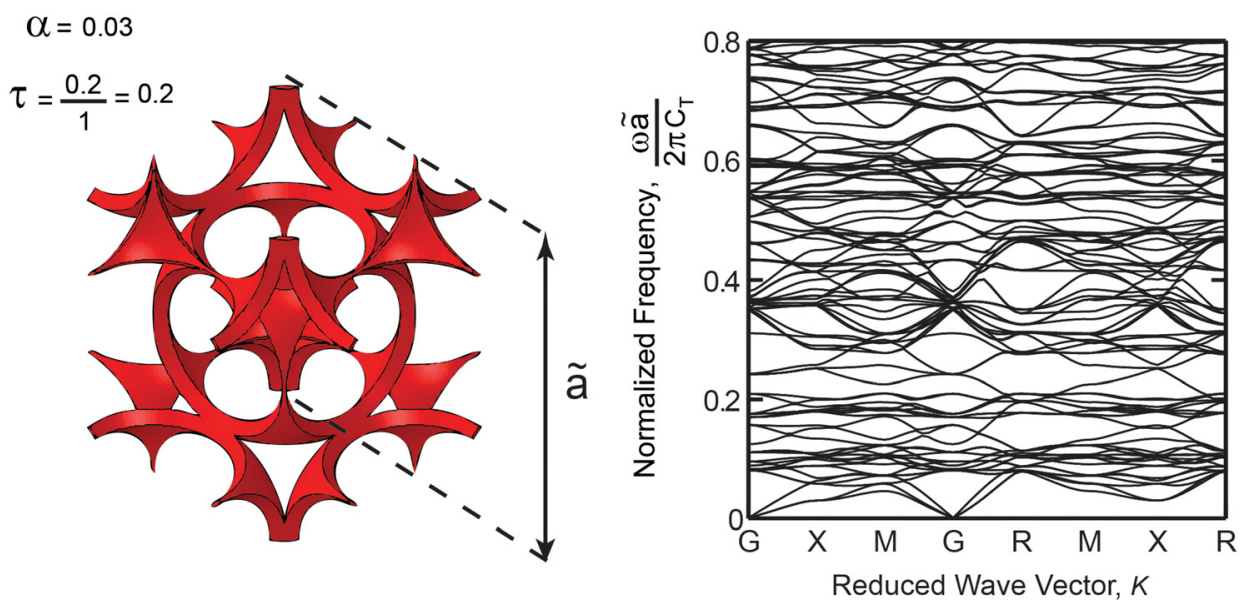

FIG. 6. Bands diagram for a phononic crystal composed of a $b c c$ array of structured shells attached to the neighbors through the ligaments. The structured shells are characterized by $(\alpha, \tau)$ $=(0.03,0.2)$. Note that these are the same design parameters that we used to construct the phononic crystal shown in Fig. 5-right. $\tilde{a}=4 r_{o} / \sqrt{2}$ is the characteristic length of the unit cell.

ligaments rather than through the corners (see Fig. 6). For this periodic structure, no bandgap is found.

Next, we systematically study the effect of the design parameters $\alpha$ and $\tau$ on the propagation of elastic waves. Since the phononic bandgaps are strongly influenced by the smallest geometric features in the system, ${ }^{48}$ a bandgap opens when $\alpha$ is decreased below a critical threshold. The effect of $\tau$ on the bandgap is instead more unpredictable, since smaller values of $\tau$ result in a structure characterized by thinner ligaments width $w_{i}$, but larger thickness $t$. Therefore, to identify the $3 \mathrm{D}$ crystal with the widest phononic bad gap in the undeformed configuration, we choose $\alpha=0.03$ and conduct a parametric study to explore the influence of $\tau$. In Fig. 7(a), we show the evolution of the bandgap as a function of $\tau$. The results clearly indicate that the width of the bandgap does not monotonically increase as a function of $\tau$ and the largest bandgap is achieved for $\tau=0.4$ (see Figs. 7(b) and 7(c)).

\section{B. Effect of deformation on wave propagation}

Having identified the optimal configuration in the undeformed configuration, we now proceed to explore the propagation of small-amplitude elastic waves in such 3D crystal at different levels of applied deformation. Here, to preserve the symmetry of the structure and facilitate the calculation of the band diagram, we compress the 3D crystal equi-triaxially. First, we perform a linear stability analysis and find that for $\tau=0.4$ buckling induces the spherical collapse of every building block 
(a)

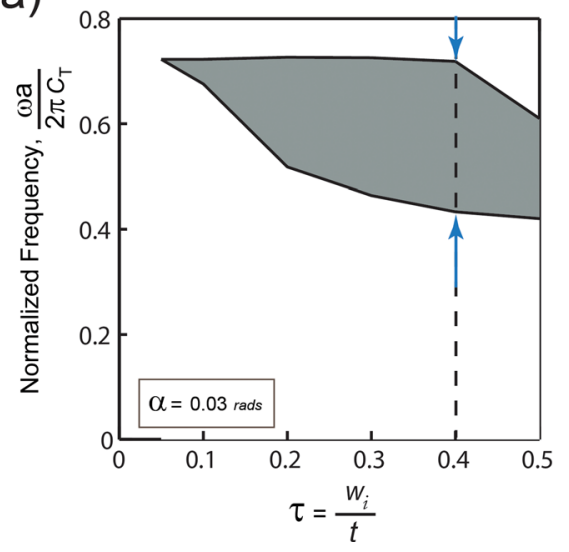

(b)

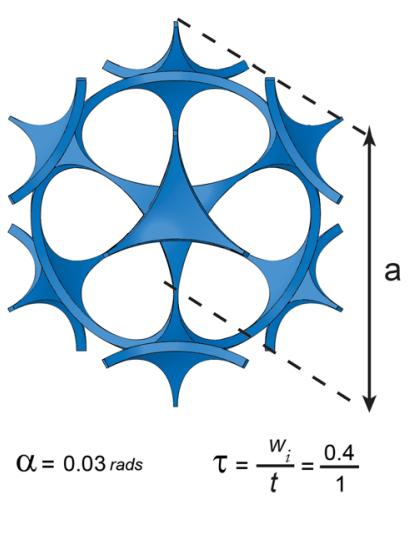

(c)

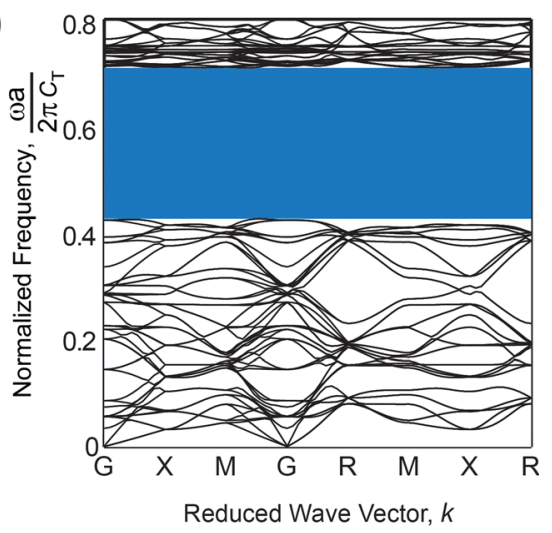

FIG. 7. Design of an optimal 3D phononic crystal in the undeformed configuration. (a) Evolution of the bandgap as a function of $\tau$ for a $3 \mathrm{D}$ crystal with $\alpha=0.03$. The structure exhibits the widest phononic bandgap for $\tau=0.4$ and is demonstrated in (b). (c) Bands diagram for the optimal crystal characterized by $(\alpha, \tau)=(0.03,0.4)$. The 3D structure exhibits a wide bandgap at $\bar{f}=0.43-0.72$.

while keeping the structure periodic (see Fig. 1). Then, we simulate the post-buckling response of the structure by introducing a small imperfection in the initial geometry and monitor the propagation of small-amplitude elastic waves as a function of the applied deformation. In Fig. 8, we report the evolution of bandgap as a function of the applied compressive strain, $\epsilon$. The results clearly indicate that the applied compression significantly alters the bandgaps by progressively reducing its width. In fact, while in the undeformed configuration the structure is characterized by a bandgap for $\bar{f}=0.43-0.72$, for $\epsilon=-0.10$ its width is reduced to $\bar{f}=0.41-0.46$. Finally, for $\epsilon=-0.16$, the bandgap completely disappears, so that elastic waves of all frequencies are able to propagate within the structure. Therefore, our results clearly demonstrate that the proposed 3D structure acts as a reversible phononic switch, since a moderate level of strain (i.e., $\epsilon=-0.16$ ) can be used to switch off the bandgap.

\section{Damped phononic crystal}

Our results indicate that mechanical instabilities and large deformation can be effectively utilized to reversibly

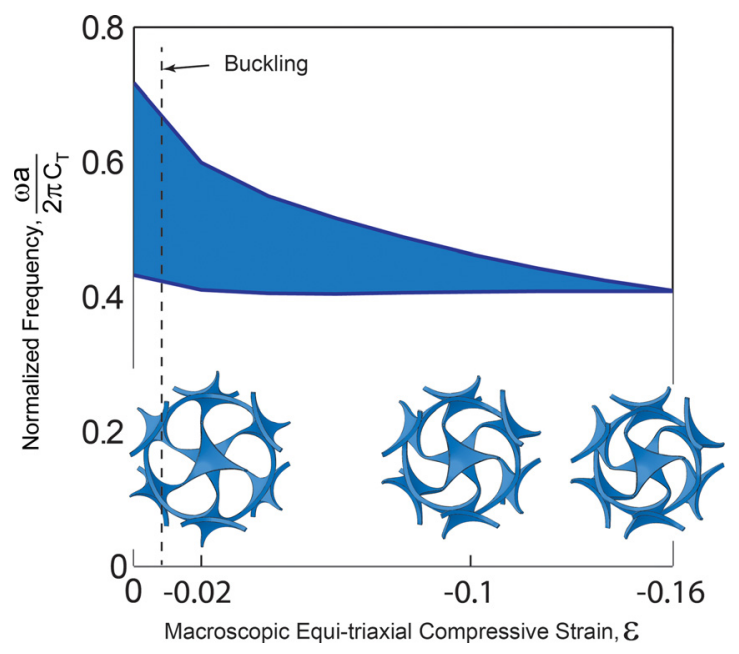

FIG. 8. Evolution of bandgap as a function of the applied compressive strain for a $3 \mathrm{D}$ crystal characterized by $(\alpha, \tau)=(0.03,0.4)$. Note that buckling occurs at $\epsilon=-0.007$. tune and control the phononic bandgaps of 3D periodic structures. However, in order for the structure to sustain the large deformation induced by buckling while remaining in the fully elastic regime, elastomeric materials such as silicon rubber are typically used for fabrication. These materials are known to be dissipative and so we now investigate the effect of material damping on the dynamic response of the $3 \mathrm{D}$ crystals.

To investigate the effect of material damping on the propagation of small-amplitude elastic waves, we start by constructing phononic crystals consists of 4, 10, 20, and 40 unit cells along the $\mathrm{x}$-direction (indicated in Fig. 1) with periodic boundary conditions on the lateral faces. In Fig. 9, we report the transmission spectra for such structures characterized by $(\alpha, \tau)=(0.03,0.4)$ and consider three different stiffnessproportional damping coefficients $(\bar{q}=0,0.005,0.02)$.

Focusing on the undamped crystals ( $\bar{q}=0$ - red lines), we find a good agreement between the drop in transmission observed in the four models (dashed horizontal red line) and the bandgap-size predicted by the Bloch wave analysis for the corresponding infinite structure (solid horizontal red line). Moreover, as expected, we find that the agreement is improved and the amount of drop in transmission is more pronounced for larger structures (i.e., $\sim-15 \mathrm{~dB}$ for the model with four unit cells in Fig. 9(a) and $\sim-25 \mathrm{~dB}$ for the model with 40 unit cells in Fig. 9(d)).

Furthermore, our results reveal that material damping has a significant effect on the transmission spectrum and that all waves with frequencies above a cutoff value are damped. To quantify this effect, the cutoff frequency, denoted by $\bar{f}_{\text {cutoff }}$, is defined as the critical frequency above which all waves are attenuated by at least $10 \mathrm{~dB}$. Such cutoff frequencies are indicated by the green and blue horizontal dashed lines in Fig. 9. The transmission response continues to decay for frequencies higher than $\bar{f}_{\text {cutoff }}$. Interestingly, we see that by increasing the size of the structure, the cutoff frequencies are progressively lowered. In fact, in a larger structure, the wave travels through a longer medium, so that the effect of dissipative medium on transmission spectrum is more pronounced for the same level of damping. However, we note that the cutoff frequencies are the same for the model with 
(a)

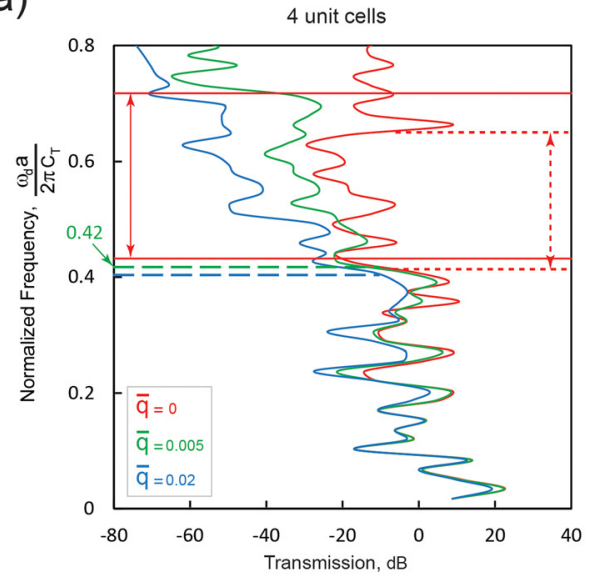

(c)

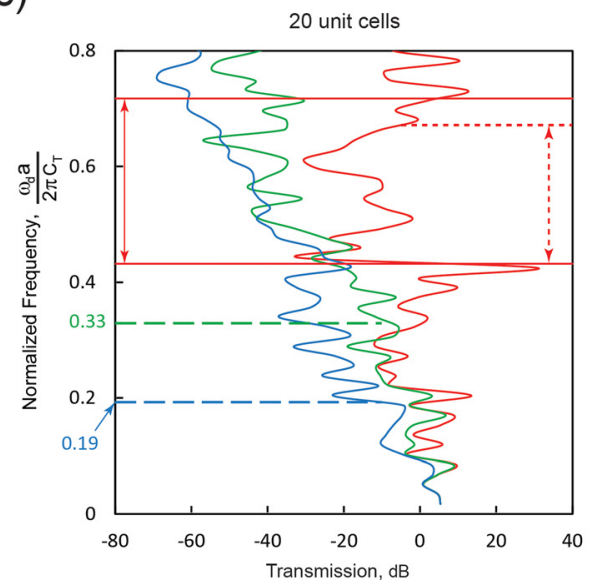

(b)

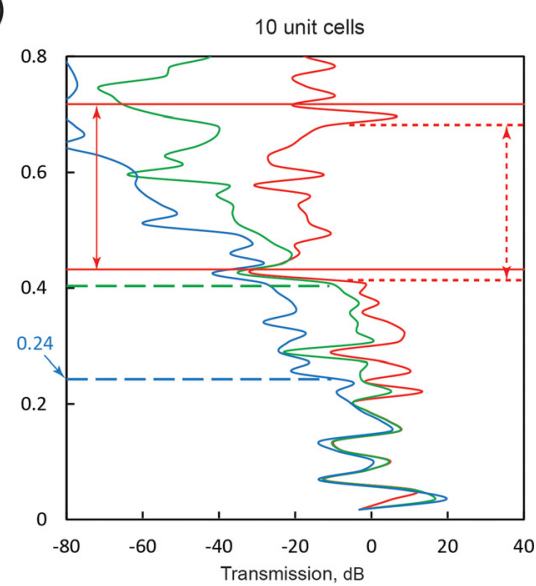

(d)

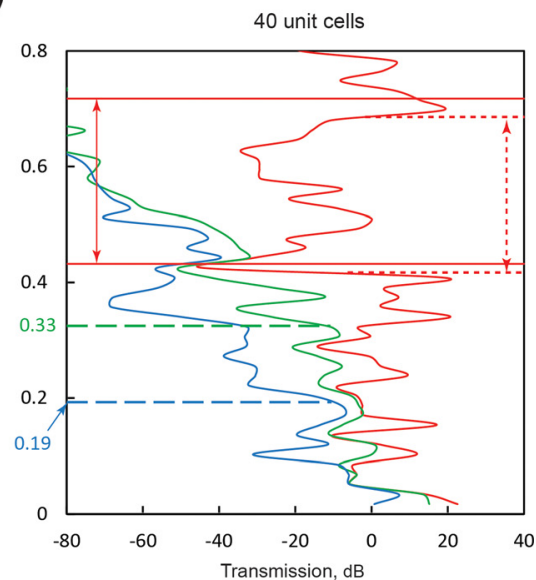

FIG. 9. Effect of damping on the transmission spectra for models consists of (a) 4, (b) 10, (c) 20, and (d) 40 unit cells along the $\mathrm{x}$-direction and periodic boundary conditions on the lateral faces. The solid horizontal red lines represent the bandgap predictions for the corresponding undamped infinite periodic structures. The horizontal dashed red lines highlight regions with a relative $-15 \mathrm{~dB}$ drop in transmission. Note that for $\bar{q}=0.005$ and 0.02 , the transmission decays above a cut-off frequency.
20 and 40 unit cells along the x-direction $\left(\bar{f}_{\text {cutoff }} \sim 0.33\right.$ for $\bar{q}=0.005$ and $\bar{f}_{\text {cutoff }} \sim 0.19$ for $\bar{q}=0.02$ ), indicating that these structures are large enough so that the dynamic response is not anymore affected by the size of the system.

Finally, in Fig. 10, we report results for a finite size structure with $4 \times 4 \times 4$ unit cells. We find that the bandgap for the undamped case (i.e., $\bar{q}=0$ - red curve) is relatively smaller than that of the corresponding infinite crystal, indicating that the finite size of the structure significantly affects its dynamic response. Moreover, we note that as we increase $\bar{q}$, material dissipation has still a significant effect, but not as pronounced as for the systems investigated in Fig. 9. In fact, we find that for $\bar{q}=0.005$, the structure is still characterized by a bandgap, highlighted by the drop in transmission at $\bar{f}=0.51-0.63$. However, for $\bar{q}=0.02$, the response is characterized by cutoff frequency and the propagation of waves with $\bar{f}>0.5$ is prevented by the dissipative medium. Therefore, these results highlight the important role played by the size of the system in the case of a damped phononic crystal.

\section{CONCLUSIONS}

In conclusion, our study outlines a general strategy in which a judicious choice of building blocks provides a
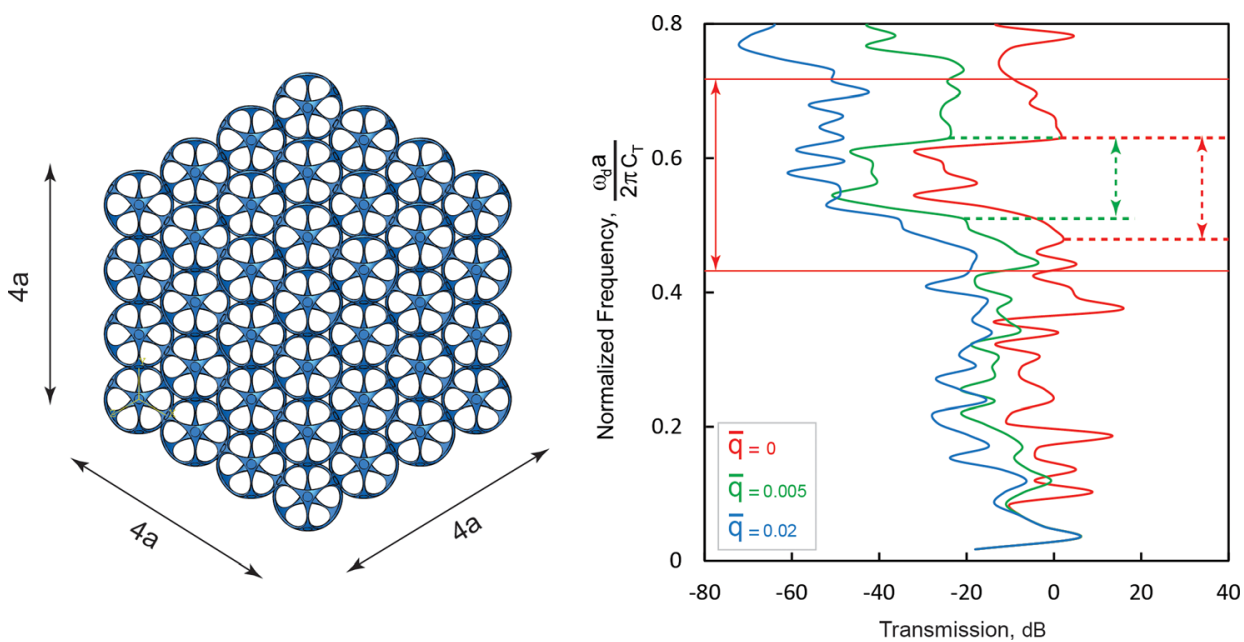

FIG. 10. Effect of damping on the propagation of elastic waves in a finite size structure consists of $4 \times 4 \times 4$ structured shells. The solid horizontal lines indicate the bandgaps prediction based on calculations for the infinite undamped structure, while the dashed horizontal lines highlight regions in which a relative $-15 \mathrm{~dB}$ drop is observed in the transmission. 
foundation for the design of 3D phononic crystals whose response can be effectively tuned by the applied deformation, opening the door to the design of $3 \mathrm{D}$ phononic switches. In particular, we show that the tunability of bandgaps can be significantly increased by exploiting the geometric nonlinearity triggered by instabilities. Moreover, we investigate the effects of energy dissipation induced by elastomeric materials on the dynamic response of 3D phononic crystals. Our results indicate that the propagation of waves in structures made of dissipative media is affected by both the amount of damping and the size of the system, providing a complete set of guidelines for the design of 3D adaptive phononic crystals.

Finally, since the buckling phenomenon is scaleindependent and the elastic deformation process is fully reversible, we expect our results will serve as a blueprint for the design of a new class of tunable phononic devices in a wide range of applications.

\section{ACKNOWLEDGMENTS}

This work has been supported by Harvard MRSEC through Grant No. DMR-1420570 and by NSF through Grant Nos. CMMI-1120724 and CMMI-1149456 (CAREER). K.B. acknowledges start-up funds from the Harvard School of Engineering and Applied Sciences and the support of the Kavli Institute and Wyss Institute at Harvard University.

${ }^{1}$ L. J. Gibson and M. F. Ashby, Cellular Solids: Structure and Properties (Cambridge University Press, 1999).

${ }^{2}$ T. Schaedler, A. Jacobsen, A. Torrents, A. Sorensen, J. Lian, J. Greer, L. Valdevit, and W. Carter, Science 334, 962 (2011).

${ }^{3}$ X. Zheng, H. Lee, T. H. Weisgraber, M. Shusteff, J. DeOtte, E. B. Duoss, J. D. Kuntz, M. M. Biener, Q. Ge, J. A. Jackson et al., Science 344, 1373 (2014).

${ }^{4}$ S. Babaee, B. H. Jahromi, A. Ajdari, H. Nayeb-Hashemi, and A. Vaziri, Acta Mater. 60, 2873 (2012).

${ }^{5}$ M. Maldovan and E. L. Thomas, Periodic Materials and Interference Lithography: For Photonics, Phononics and Mechanics (Wiley-VCH Verlag GmbH \& Co. KGaA, 2009).

${ }^{6}$ Acoustic Metamaterials and Phononic Crystals, edited by P. A. Deymier (Springer, 2013).

${ }^{7}$ M. Sigalas and E. N. Economou, Solid State Commun. 86, 141 (1993).

${ }^{8}$ M. Kushwaha, P. Halevi, L. Dobrzynski, and B. Djafari-Rouhani, Phys. Rev. Lett. 71, 2022 (1993).

${ }^{9}$ M. I. Hussein, M. J. Leamy, and M. Ruzzene, Appl. Mech. Rev. 66, 040802 (2014).

${ }^{10}$ Z. Liu, X. Zhang, Y. Mao, Y. Zhu, Z. Yang, C. Chan, and P. Sheng, Science 289, 1734 (2000)

${ }^{11}$ T. Still, W. Cheng, M. Retsch, R. Sainidou, J. Wang, U. Jonas, N. Stefanou, and G. Fytas, Phys. Rev. Lett. 100, 194301 (2008).

${ }^{12}$ I. Psarobas, A. Modinos, R. Sainidou, and N. Stefanou, Phys. Rev. B 65, 064307 (2002).
${ }^{13}$ A. Bretagne, B. Venzac, V. Leroy, and A. Tourin, AIP Conf. Proc. 1433, 317-318 (2012).

${ }^{14}$ V. K. Kinra and E. L. Ker, Int. J. Solids Struct. 19, 393 (1983).

${ }^{15}$ M. Kafesaki, M. Sigalas, and E. Economou, Solid State Commun. 96, 285 (1995).

${ }^{16}$ X. Zhang, Z. Liu, Y. Liu, and F. Wu, Phys. Lett. A 313, 455 (2003).

${ }^{17}$ J. Page, S. Yang, M. Cowan, Z. Liu, C. Chan, and P. Sheng, Wave Scattering in Complex Media: From Theory to Applications, NATO Science Series Vol. 107, edited by B. van Tiggelen and S. Skipetrov (Springer, Netherlands, 2003), pp. 282-307.

${ }^{18}$ S. Yang, J. Page, Z. Liu, M. Cowan, C. Chan, and P. Sheng, Phys. Rev. Lett. 93, 024301 (2004).

${ }^{19}$ R. Sainidou, B. Djafari-Rouhani, Y. Pennec, and J. O. Vasseur, Phys. Rev. B 73, 024302 (2006).

${ }^{20}$ C. Daraio, Ph.D. thesis (University of California, San Diego, 2006).

${ }^{21}$ L. Wang and K. Bertoldi, Int. J. Solids Struct. 49, 2881 (2012).

${ }^{22}$ J. Jang, C. K. Ullal, T. Gorishnyy, V. V. Tsukruk, and E. L. Thomas, Nano Lett. 6, 740 (2006).

${ }^{23}$ K. Bertoldi and M. Boyce, Phys. Rev. B 78, 184107 (2008).

${ }^{24}$ P. Wang, J. Shim, and K. Bertoldi, Phys. Rev. B 88, 014304 (2013).

${ }^{25}$ S. Rudykh and M. C. Boyce, Phys. Rev. Lett. 112, 034301 (2014).

${ }^{26}$ S. Shan, S. H. Kang, P. Wang, C. Qu, S. Shian, E. R. Chen, and K. Bertoldi, Adv. Funct. Mater. 24, 4935 (2014).

${ }^{27}$ D. Mousanezhad, S. Babaee, R. Ghosh, E. Mahdi, K. Bertoldi, and A. Vaziri, "Honeycomb phononic crystals with self-similar hierarchy," Phys. Rev. Lett. (submitted).

${ }^{28}$ P. Wang, F. Casadei, S. Shan, J. C. Weaver, and K. Bertoldi, Phys. Rev. Lett. 113, 014301 (2014).

${ }^{29}$ S. Babaee, J. Shim, J. C. Weaver, E. R. Chen, N. Patel, and K. Bertoldi, Adv. Mater. 25, 5044 (2013).

${ }^{30}$ J. Shim, C. Perdigou, E. R. Chen, K. Bertoldi, and P. M. Reis, Proc. Natl. Acad. Sci. U.S.A. 109, 5978 (2012).

${ }^{31}$ R. W. Ogden, Non-Linear Elastic Deformations (Dover, 1997).

${ }^{32}$ L. Treloar, Trans. Faraday Soc. 40, 59 (1944).

${ }^{33}$ G. Geymonat, S. Müller, and N. Triantafyllidis, Arch. Ration. Mech. Anal. 122, 231 (1993).

${ }^{34}$ K. Bertoldi, M. Boyce, S. Deschanel, S. Prange, and T. Mullin, J. Mech. Phys. Solids 56, 2642 (2008).

${ }^{35}$ F. Bloch, Z. Phys. 52, 555 (1929).

${ }^{36}$ L. Brillouin, Wave Propagation in Periodic Structures (McGraw-Hill, 1946).

${ }^{37}$ M. Maldovan and E. Thomas, Periodic Materials and Interference Lithography for Photonics, Phononics and Mechanics (Wiley-VCH, 2009).

${ }^{38}$ M. I. Hussein, Proc. R. Soc. A 465, 2825 (2009).

${ }^{39}$ T. K. Caughey and M. E. J. O'Kelly, J. Appl. Mech. 32, 583 (1965).

${ }^{40} \mathrm{~J}$. W. Rayleigh, The Theory of Sound, 2nd ed. (Macmillan and Co., London and New York, 1894), Vol. 1.

${ }^{41} \mathrm{~W}$. Thomason and M. Dahleh, Theory of Vibration With Applications, 5th ed. (Prentice Hall, 1998).

${ }^{42}$ M. I. Hussein and M. J. Frazier, J. Appl. Phys. 108, 093506 (2010).

${ }^{43}$ M. I. Hussein, Phys. Rev. B 80, 212301 (2009).

${ }^{44}$ D. Koblar and M. Boltežar, Exp. Tech. 2013, 1-10; available at http:// onlinelibrary.wiley.com/doi/10.1111/ext.12066/abstract.

${ }^{45} \mathrm{~V}$. Adams and A. Askenazi, Building Better Products With Finite Element Analysis, 1st ed. (OnWord Press, Santa Fe, NM, 1999).

${ }^{46}$ D. J. Ewins, Modal Testing: Theory, Practice and Application, 2nd ed. (Research Studies Press Ltd, Baldock, Hertfordshire, UK, 2000).

${ }^{47}$ Abaqus Analysis User's Manual Version 6.11, Dassault Systemes Simulia Corp., Providence, RI, 2012.

${ }^{48}$ Z. Liu, C. Chan, and P. Sheng, Phys. Rev. B 65, 165116 (2002). 